CIRJE-F-1112

\title{
A Two-Stage Model of Assignment and Market
}

\author{
Akihiko Matsui \\ The University of Tokyo \\ Megumi Murakami \\ Northwestern University
}

January 2019

CIRJE Discussion Papers can be downloaded without charge from:

http://www.cirje.e.u-tokyo.ac.jp/research/03research02dp.html

Discussion Papers are a series of manuscripts in their draft form. They are not intended for circulation or distribution except as indicated by the author. For that reason Discussion Papers may not be reproduced or distributed without the written consent of the author. 


\title{
A TWO-STAGE MODEL OF ASSIGNMENT AND MARKET
}

\author{
AKIHIKO MATSUI AND MEGUMI MURAKAMI
}

\begin{abstract}
Aвstract. Centralized matching mechanisms and decentralized markets have been widely studied to allocate indivisible objects. However, they have been analyzed separately. The present paper proposes a new framework, by explicitly formulating a two-stage model where objects are allocated through a matching mechanism in the first stage and traded in the second stage market. In addition, one divisible good called money may or may not be available in the market. Every player demands at most one unit of object besides money. The players may face different priorities at each object type in the first stage. Each object type has a limited amount of capacity, called quota. Each player has a quasi-linear utility function. The present analysis sets forth the equivalence conditions under which stability and efficiency are attained in equilibrium.
\end{abstract}

Keywords: two-stage economy, deferred acceptance algorithm (DA), market, indivisible object, perfect market equilibrium (PME), priority, stability, cyclical priority, unreversed priority, minimal demand

JEL Classification Numbers: C78, D41, D47, D51

\section{INTRODUCTION}

Centralized matching mechanisms and decentralized markets have been widely studied to assign indivisible objects. On the one hand, matching mechanisms have been studied since the seminal paper of Gale and Shapley (1962). A class of problems investigated therein include college admission problems, housing allocations and office assignments. In these problems, a centralized mechanism assigns objects to participants based on their preferences over objects and priorities over individuals.

On the other hand, the markets of indivisible objects have been analyzed since Shapley and Shubik (1971). Housing and labor markets are typical problems analyzed in this literature. Given the ownership of the objects, demand and supply meet by way of price.

Although a matching mechanism and a market have been analyzed separately, the former is often followed by the latter in reality, and choices in the matching mechanism are affected by the subsequent market. Take college admission as an example. Students do not necessarily choose

Date: January 24, 2019.

Incomplete.

We are grateful to the participants of seminars at Keio University, Kyoto University, Kobe University, National University of Singapore, Northwestern University, Okayama University, UT Summer School in Economics, and Waseda University as well as In-Koo Cho, Ryota Iijima, Mamoru Kaneko, Daiki Kishishita, Fuhito Kojima, Shunya Noda, Wojciech Olszewski, Marciano Siniscalchi, Bruno Strulovici, and Yuki Takagi for useful conversations and comments. Financial support from the Grants-in-Aid for Scientific Research for the Promotion of Science is gratefully acknowledged. Any opinions, findings, and conclusions or recommendations expressed in this material are those of the authors and do not necessarily reflect the views of the Japan Society for the Promotion of Science. 
a college based on its own characteristics such as campus and curriculum. Rather, they take the future job prospects upon graduation into consideration. In other words, the students solve a twostage problem, the college admission problem followed by that of the job market.

Airport coordination is another example of the two-stage model. This is a mechanism to allocate airport slots, permissions to use the full range of airport infrastructure to arrive or depart at some airports on a specific date and time. International Air Transport Association (IATA) sets Worldwide Slot Guidelines to allocate the limited number of slots efficiently. According to the guideline, the first stage is a centralized assignment. Each airline submits its preferences over the slots. Coordinators and facilitators decide the slot assignments based on the priorities determined by the guideline. In the second stage after the initial allocation, the Slot Conference is held to adjust or exchange the assigned slots. According to IATA, this conference is "attracting over 1200 delegates, from over 230 airlines and representatives of over 85 schedules-facilitated or fully coordinated airports" "to discuss the arrangement of flight schedules. At the conference, adjustments are proceeded mainly through the bilateral meeting between a coordinator and an airline delegate. After the conference, final schedules are determined.

The existing single-stage models can be viewed as the reduced forms of the two-stage problems. It is not obvious, however, that the properties of the reduced single-stage models correspond to those of the full-fledged two-stage models. The present analysis shows that they do not coincide with each other in general.

The present paper proposes a new framework, by explicitly formulating a two-stage model where indivisible objects are allocated through the first stage matching mechanism and traded in the second stage market. In this model, there are finitely many players and finitely many types of indivisible objects. In addition, one divisible good called money may or may not be available in the market. Every player demands at most one unit of object besides money. The players may face different priorities at each object type in the first stage. Each object type has a limited amount of capacity, called quota. Each player has a quasi-linear utility function. There is an object type called the null object, which induces zero value for all the players and has a sufficient amount of quota. An object type other than the null object is called a tangible object type.

The first stage is governed by a non-monetary assignment mechanism. The present paper uses the deferred acceptance algorithm (DA) of Gale and Shapley (1962). The participants simultaneously submit the lists of their preferences, and objects are allocated through DA.

The second stage is governed by the market. The objects assigned in the first stage become the endowments of the second stage. The priority no longer matters in the second stage since every available object is owned by some player. The players trade objects in the market. The present paper considers both non-monetary and monetary markets. In the airport coordination problem, the Slot Conference implements non-monetary exchanges, while the job market after college admission involves monetary contracts. Each player's payoff is determined by the indivisible object and money, if any, held at the end of the second stage. In particular, what they obtain in the first stage matter only to the extent that these objects affect the final allocation of the second stage.

The set of the participants in DA may be strictly contained in the entire set of the players. In the college admissions problem with the subsequent labor market, for example, the entire players are divided into two, students and firms, and only the students participate in the college admission stage.

${ }^{1}$ URL: https: //www . iata.org/events/sc144/Pages/index . aspx, retrieved on Jan. 24, 2019. 
As an equilibrium concept, perfect market equilibrium (PME) is defined. PME requires that a market equilibrium should be realized in each market of the second stage both on and off the paths, and that each player should select an optimal strategy in the first stage mechanism, taking the second stage outcome into consideration. To be precise, we define an induced game, where the payoff of each strategy profile is defined by the corresponding market equilibrium outcome. A PME is a Nash equilibrium of this induced game.

First of all, the existence of PME is shown. In the economies with no money, a PME exists if the values of tangible objects are positive for all the players, and if the quota of each tangible object is one ${ }^{2}$. These conditions are assumed to hold in the economies with no money. In the economies with money, a PME always exists in the present framework.

Next, other properties are studied. In the economies with no money, the main question is when Pareto optimality and the stability of PME allocations are attained. First, an allocation is Pareto optimal if no player cannot be better off without reducing the other players' payoffs. Second, an allocation is stable if every player prefers an object to her own object only if the object is assigned to the player who has higher priority than her at the object.

On the one hand, Gale and Shapley (1962) shows that the allocation induced by the truth-telling strategy profile in the single-stage DA mechanism, called the DA allocation hereafter, is always stable, while Ergin (2002) shows that the DA allocation is Pareto optimal if and only if the priority profile is acyclical.

On the other hand, the present paper shows that any pure PME allocation is always Pareto optimal, while the PME allocation with the truth-telling strategy profile is stable if and only if the priority profile is acyclical. Moreover, the PME allocation coincides with the DA allocation. This relationship is summarized in Table 1.1.

\begin{tabular}{|c|c|c|}
\hline Allocation & Pareto Optimality & Stability \\
\hline DA & iff $>$ is acyclical (Ergin) & always (Gale and Shapley) \\
\hline PME with TT & always & iff $>$ is acyclical \\
\hline
\end{tabular}

TABLE 1.1

However, even if the priority profile is acyclical, there may exist a PME of which allocation is not stable. This leads to the next condition. To begin with, two distinct players are said to be top-two players if the priority of each of these two players is higher than any other player at every object type. We then say that priority profile is unreversed if all the players except for these top-two players, if any, have the same priority order across the object types. It is shown that the Pareto optimal DA allocation is the unique pure PME allocation if and only if the priority profile is unreversed.

In the economies with money, the question is when PME allocations attain efficiency. An efficient allocation maximizes the sum of the players' utility values among all the feasible object allocations. Two cases are considered. In the first case, the players may become buyers and sellers at the same time. The present analysis sets forth the equivalence conditions under which efficiency is attained in a PME. It is shown that the efficient object allocation is uniquely achieved in a PME

\footnotetext{
${ }^{2}$ It is shown, by counterexample, that a PME does not necessarily exist if one of these conditions is violated.
} 
if and only if the minimal demand, the number of players that put positive values to each object, exceeds a certain threshold.

In the second case, players are divided into two groups, sellers and buyers. This analysis may be interpreted as a college admission problem followed by a labor market: the objects are college degrees, the sellers are students, and the buyers are firms. The first stage is a many-to-one matching between students and colleges. In the second stage, a job market opens, and each firm hires at most one student based on his/her college degree ${ }^{3}$. It is shown that the efficient allocation is uniquely achieved in a PME if and only if both the number of the firms with positive values and the number of the students exceed certain thresholds.

The first stage of the present model is based upon the literature on DA. The college admission problem is adopted from Gale and Shapley (1962) and Roth and Sotomayor (1989). The model of the present paper formulates the first stage as a game. This formulation is taken from Sotomayor (2008). We use the result of the strategy-proofness of DA by Dubins and Freedman (1981) and Roth (1982). Ergin (2002) shows the equivalence between the efficiency of the allocation induced by DA and the acyclicity of the priority profile, which is key to proving one of the main results of the present paper.

The second stage of the present model is based upon the literature on markets with indivisible objects. Shapley and Scarf (1974) shows non-emptiness of core and existence of competitive equilibrium when there is no money. The present analysis uses their result directly in proving the existence of market equilibrium in the case of no money. Kaneko and Yamamoto (1986) proves the existence of competitive equilibrium in an economy with indivisible objects and money ${ }^{4}$. The present paper uses this result directly in showing the existence of market equilibrium in the case with money.

If we view the first stage as the assignment stage of property rights, then the analysis of the present paper is related to the literature on property rights' assignment with resale and mechanism design with renegotiation, which is abundant. First of all, it is related to Coase's theorem (see Coase (1960)). In the present context, the theorem implies that irrespective of the assignment of property right, the market leads to an efficient allocation. Jehiel and Moldovanu (1999) considers assignment with resale and shows that the assignment of property right is irrelevant if there are resale processes. Their result corresponds to the result of the present paper in the case with money when there is a sufficient amount of demand for the objects. If the demand is insufficient, however, some objects may remain unassigned, and therefore, inefficiency is induced. Hafalir and Krishna (2008) considers a two-stage model where auction is held in the first stage, and the players who obtain the objects through the auction trade them in the second stage. The present model is different from Hafalir and Krishna (2008) in that the first stage mechanism is DA, while theirs is auction. Maskin and Moore (1999) considers a two-stage model where a mechanism is implemented in the first stage, but the players cannot commit to its outcome in the second stage and may renegotiate to move to a Pareto-improving outcome. The present paper studies a specific environment to obtain equivalence results.

\footnotetext{
${ }^{3}$ Crawford and Knoer (1981) considers a model in which matching between firms and workers are adjusted by a centralized mechanism that is an extension of DA by Gale and Shapley (1962). In contrast, the present model assumes that workers and firms participate in the discentralized market.

${ }^{4}$ Kaneko (1982) proves non-emptiness of core under non-transferable utility. Wako (1984) shows that strong core is inside the set of competitive equilibrium and demonstrates the conditions under which strong core exists.
} 
The rest of the paper is organized as follows. Section 2 presents a model and a solution concept as well as some preliminary results. Section 3 studies economies without money. Section 4 studies economies with money where sellers and buyers are not necessarily separated. Section 5 studies an economy where the population is divided into two groups, sellers and buyers. Section 6 concludes the paper. Some proofs and examples are relegated to appendices.

\section{MODEL}

A two-stage economy is considered. In the first stage, players participate in an assignment game to obtain objects, while in the second stage, a market opens to allocate objects and money, if any. The assignment of the objects in the first stage is governed by the deferred acceptance algorithm (DA). The second stage is a pure exchange economy with the initial endowments being the outcome of the first stage.

2.1. Preliminaries. $N$ is a finite set of players. $O$ is a finite set of object types. Assume $|N| \geq 2$ and $|O| \geq 2$. There is a null object type, denoted $\phi$. We may call $\phi$ an object and any $a$ in $O$ a tangible object whenever convenient. Let $\bar{O}=O \cup\{\phi\}$. For any $a \in O$, $a$ has a quota $q^{a} \in\{1,2, \ldots\}$. Also, let $q^{\phi}$ be a pseudo quota for $\phi$. We assume $q^{\phi}=|N|$. A quota profile is denoted by $q=\left(q^{a}\right)_{a \in O}$. Let $Q=\sum_{a \in O} q^{a}$. The objects are indivisible, and each player demands at most one unit of object. Given a vector $\mu=\left(\mu_{i}\right)_{i \in N} \in \bar{O}^{N}$ and $a \in \bar{O}$, let $\mu^{a}=\left\{i \in N \mid \mu_{i}=a\right\}$ be the set of the players who hold $a$.

Two classes of economies, one with no money and the other with money, are considered. The latter allows the players to use divisible money for the second stage transaction in addition to the objects, while the former does not.

An object allocation is $\mu \in \bar{O}^{N}$ that satisfies $\left|\mu^{a}\right| \leq q^{a}$ for all $a \in O$. If no money is available, an allocation is given by $\mu$. If money is available, the initial money holding is zero for every player, while the eventual money holding of each player can be positive or negative. A monetary allocation is $m \in \mathbb{R}^{N}$ with $\sum_{i \in N} m_{i}=0$. An allocation is given by $(\mu, m)$ in the case of the economy with money. In the sequel, an allocation with no money is sometimes written as $(\mu, m)$ with the understanding that $m=(0, \ldots, 0)$ holds.

Utility functions are assumed to be quasi-linear, i.e., for every $i \in N$, the utility function $u_{i}$ : $\bar{O} \times \mathbb{R} \rightarrow \mathbb{R}$ of agent $i$ is given by

$$
u_{i}\left(a_{i}, m_{i}\right)=v_{i}\left(a_{i}\right)+m_{i} .
$$

In the case of no money, we interchangeably write $u_{i}\left(a_{i}\right)=u_{i}\left(a_{i}, 0\right)=v_{i}\left(a_{i}\right)$. We assume $v_{i}(\phi)=0$ for every $i \in N$. The values of the tangible objects are assumed to be generic ${ }^{56}$. Let $V$ be the set of all the generic value profiles with $v_{i}(\phi)=0(i \in N)$.

\footnotetext{
${ }^{5}$ Although the subsequent examples do not satisfy genericity, their results do not essentially depend upon nongenericity.

${ }^{6}$ In particular, for all subsets $M, M^{\prime}$ of $N$ and all allocations $\mu, \mu^{\prime}$, we have $\sum_{i \in M} v_{i}\left(\mu_{i}\right) \neq \sum_{j \in M^{\prime}} v_{j}\left(\mu_{j}^{\prime}\right)$ if (at least) one of the following conditions holds: (i) $\mu_{i} \neq \phi$ for some $i \in M \backslash M^{\prime}$; (ii) $\mu_{j}^{\prime} \neq \phi$ for some $j \in M^{\prime} \backslash M$; (iii) $\mu_{i} \neq \mu_{i}^{\prime}$ for some $i \in M \cap M^{\prime}$.
} 
2.2. The first stage: assignment. In the first stage, the players obtain objects based on priority through the deferred acceptance algorithm (DA). Let $P \subset N$ be the set of participants of DA; players in $N \backslash P$ are not allowed to participate in DA. It is assumed that $|P|>q^{a}$ holds for all $a \in O$. Each participant's strategy is a list of objects ordered from the top to the bottom, i.e., for each $i \in P$, player $i$ 's list is given by $\left(a_{i}^{1}, \ldots, a_{i}^{|\bar{O}|}\right)$. The non-participants are dummy players: their unique strategy is $\phi$. Let $\Sigma_{i}(i \in N)$ be the set of $i$ 's strategies. Let $\Sigma=\left(\Sigma_{i}\right)_{i \in N}$ be the set of strategy profiles and $\lambda: \Sigma \rightarrow \bar{O}^{N}$ be an outcome function, where $\lambda_{i}(\sigma)$ is given by DA for each $i \in P$, and $\lambda_{i}(\sigma)=\phi$ holds for each $i \in N \backslash P$.

For every $a \in O,>_{a}$ is a strict total order at $a \in O$ over $N$, i.e., it satisfies transitivity and asymmetry, and has no non-comparable pairs ${ }^{7}$. It defines the order of players' priority at object $a$, i.e., $i>_{a} j$ means that $i$ has higher priority than $j$ at $a$. Let $>=\left(>_{a}\right)_{a \in O}$ be a priority profile. Note that priority is defined not only over participants but also over non-participants since we change the set of participants for a fixed priority profile.

The rest is determined by the algorithm.

Step 1: Start with $\left(a_{i}^{1}\right)_{i \in P}$, the first objects of the players' respective lists.

$\star$ For each $a \in \bar{O}$, if the number of the players choosing $a$ does not exceed $q^{a}$, then they are temporarily assigned to $a$.

* If the number of the players choosing $a$ exceeds $q^{a}$, then the top $q^{a}$ players in terms of priority are temporarily assigned to $a$, and the rest go to the next step with the second objects in their respective lists.

Step $t(t>1)$ : Those assigned to $a$ before and those who choose $a$ in this step compete for $a$, and repeat $\star$ 's in Step 1 where we replace the second objects with the $(t+1)$ th objects.

Terminate the process when all the participants are assigned to an object in $\bar{O}$.

Let $A$ be the set of outcomes of the first stage. Note that each $\omega$ in $A$ satisfies $\omega_{i}=\phi$ for $i \in N \backslash P$.

2.3. The second stage: market. All the players enter the market in the second stage. Their endowment profile $\omega \in A$ is the outcome of the first stage. Tangible objects that are not assigned to any player in the first stage cannot be allocated in the second stage.

Given $\omega \in A$ and $a \in O$, let $\left|\omega^{a}\right|$ be the total endowment of object $a$ in the second stage. We denote a total endowment profile, or simply a total endowment, by $|\omega|=\left(\left|\omega^{a}\right|\right)_{a \in O}$. Given an initial object allocation $\omega \in A$ of the second stage, an allocation $(\mu, m)$ is feasible under $\omega$ if for all $a \in O$, $\left|\mu^{a}\right| \leq\left|\omega^{a}\right|$ holds. $A^{\omega}$ denotes the set of feasible allocations under $\omega$. Also, $O^{\omega}=\left\{a \in O|| \omega^{a} \mid>0\right\}$ is the set of feasible object types, and $\bar{O}^{\omega}=O^{\omega} \cup\{\phi\}$. Note that the quantity restriction is only on the objects in $O$, i.e., not on $\phi$.

The solution concept used for the second stage is market equilibrium.

Definition 2.1. Given $\omega \in A$, $(p,(\mu, m)) \in \mathbb{R}_{+}^{\bar{O}^{\omega}} \times A^{\omega} \times \mathbb{R}^{N}$ is a market equilibrium under $\omega$ if $p_{\phi}=0$ holds, and

- $\left(\mu_{i}, m_{i}\right) \in \arg \max _{\left(\mu_{i}^{\prime}, m_{i}^{\prime}\right)} u_{i}\left(\mu_{i}^{\prime}, m_{i}^{\prime}\right)$ s.t. $p_{\mu_{i}^{\prime}}+m_{i}^{\prime} \leq p_{\omega_{i}}$,

- $\forall a \in O^{\omega}\left[\left|\mu^{a}\right| \leq\left|\omega^{a}\right|\right] \wedge\left[\left|\mu^{a}\right|<\left|\omega^{a}\right| \Rightarrow p_{a}=0\right]$.

Note that money holdings are always zero in the case of no money. Note also that Definition 2.1 together with feasibility under $\omega$ implies that the objects in $O$ are free disposal.

\footnotetext{
${ }^{7} \mathrm{~A}$ binary relation $>_{a}$ over $N$ is said to have no non-comparable pairs if $i \neq j$ implies either $i>_{a} j$ or $j>_{a} i$.
} 
2.4. The two-stage economy and perfect market equilbirium. The two stages are combined. First, an induced game is introduced. For each $\omega \in A$, let $(p(\omega),(\mu(\omega), m(\omega)))$ be a pair of a price vector and an allocation under $\omega$. Given a strategy profile $\sigma \in \Sigma$ and a profile $(p(\omega),(\mu(\omega), m(\omega)))_{\omega \in A}$, player $i$ 's induced payoff is given by

$$
\tilde{u}_{i}(\sigma)=u_{i}\left(\mu_{i}(\lambda(\sigma)), m_{i}(\lambda(\sigma))\right) .
$$

An induced game $\Gamma$ is a profile $\left\langle N, \Sigma,\left(\tilde{u}_{i}\right)_{i \in N}\right\rangle$. The payoff function $\tilde{u}_{i}$ is extended to the mixed strategy space where the expected utility is used. We denote by $\rho_{i} \in \Delta\left(\Sigma_{i}\right)$ a mixed strategy of player $i$ where $\Delta\left(\Sigma_{i}\right)$ is a set of probability distributions over $\Sigma_{i}$.

Now, we present an equilibrium concept that reflects the idea of perfection.

Definition 2.2. A profile $\left(\rho,(p(\omega),(\mu(\omega), m(\omega)))_{\omega \in A}\right)$ is a perfect market equilibrium (PME) if

- for all $\omega \in A,(p(\omega),(\mu(\omega), m(\omega)))$ is a market equilibrium under $\omega$;

- $\rho \in \times_{i \in N} \Delta\left(\Sigma_{i}\right)$ is a Nash equilibrium of the induced game $\left\langle N, \Sigma,\left(\tilde{u}_{i}\right)_{i \in N}\right\rangle$.

Given a PME, we sometimes call its on-path (object) allocation a PME (object) allocation.

2.5. Pareto optimality and social welfare. A feasible object allocation is limited by the number of the participants of the first stage. Also, in the second stage, tangible objects may be traded to non-participants. Incorporating these points, we let

$$
\bar{A}=\cup_{\omega \in A} A^{\omega}
$$

be the set of all the feasible object allocations in the second stage. Then, we let

$$
X=\bar{A} \times\left\{m \in \mathbb{R}^{N} \mid \sum_{i \in N} m_{i}=0\right\}
$$

be the set of all the feasible allocations in the second stage. Note again that the money holdings are zero in the case of no money.

Two criteria are used to evaluate allocations in terms of utility. One is Pareto criterion and the other is social welfare. Consider two allocations $(\mu, m)$ and $\left(\mu^{\prime}, m^{\prime}\right)$ in $X$. The allocation $(\mu, m)$ is said to Pareto dominate $\left(\mu^{\prime}, m^{\prime}\right)$ if for all $i \in N, u_{i}\left(\mu_{i}, m_{i}\right) \geq u_{i}\left(\mu_{i}^{\prime}, m_{i}\right)$ holds, and for some $j \in N$, $u_{j}\left(\mu_{j}, m_{j}\right)>u_{j}\left(\mu_{j}^{\prime}, m_{j}\right)$ holds. The allocation $(\mu, m) \in X$ is Pareto optimal if there is no allocation in $X$ that Pareto dominates $(\mu, m)$.

The second criterion is social welfare. For each allocation $(\mu, m)$, a social welfare is given by $W(\mu)=\sum_{i \in N} v_{i}\left(\mu_{i}\right)$. We say that $(\mu, m)$ is efficient if $\mu \in \arg \max _{\mu^{\prime} \in \bar{A}} W\left(\mu^{\prime}\right)$ holds.

Given an initial object allocation $\omega \in A$ of the second stage, an allocation $(\mu, m)$ is Pareto optimal under $\omega$ if there does not exist a feasible allocation $\left(\mu^{\prime}, m^{\prime}\right)$ under $\omega$ that Pareto dominates $(\mu, m)$. Also, an allocation $(\mu, m)$, or simply $\mu$, is efficient under $\omega$ if there does not exist a feasible allocation $\left(\mu^{\prime}, m^{\prime}\right)$ under $\omega$ such that $W\left(\mu^{\prime}\right)>W(\mu)$ holds.

Note that if $|\omega|=q$ holds, then $\bar{A}=A^{\omega}$ holds, and therefore, a Pareto optimal (resp. efficient) allocation under $\omega$ is also Pareto optimal (resp. efficient).

\section{The Economy with No Money}

This section considers the economy with no money, i.e., money holdings are always zero. It is assumed that all the tangible objects are valuable for all the players. It is also assumed that the 
quota of each tangible object in $O$ is one. These assumptions are expressed in the following.

(+Value): for all $i \in N$ and for all $a \in O, v_{i}(a)>0$,

(Quota1): for all $a \in O,\left|q^{a}\right|=1$.

Under (+Value), all tangible objects have positive intrinsic values for all the players. The set of positive value profiles is given by

$$
V_{+}=\left\{v \in V \mid \forall i \in N \forall a \in O v_{i}(a)>0\right\} .
$$

3.1. Existence. The condition for the existence of market equilibrium in the second stage is nontrivial in the case of no money. Shapley and Scarf (1974) essentially shows that for any initial endowment profile, a market equilibrium exists if (+Value) and (Quota1) hold.

Lemma 3.1. Assume (+Value), and (Quota1). For all $\omega \in A$, a market equilibrium exists under $\omega$.

The proof of this lemma is the same as that of Shapley and Scarf (1974). The existence of ME is not guaranteed if either (+Value) or (Quota1) is violated. See counter-examples in Appendix A.1.

Given the initial endowment profile $\omega \in A$ of the second stage, transactions occur in the form of trading cycles. A trading cycle is a cycle of pairs of the form:

$$
\left(\left(i_{1}, \omega_{i_{1}}\right),\left(i_{2}, \omega_{i_{2}}\right), \ldots,\left(i_{K}, \omega_{i_{K}}\right)\right)
$$

with $\left(i_{1}, \omega_{i_{1}}\right)=\left(i_{K}, \omega_{i_{K}}\right)$ such that $i_{k-1}$ trades $\omega_{i_{k-1}}$ for $\omega_{i_{k}}(k=2, \ldots, K)$ in the second stage.

Using Lemma 3.1 and the existence result of subgame perfect equilibrium for a finite game, we have the existence result for PME, which is stated without proof.

Theorem 3.2. Assume (+Value) and (Quotal). Then, there exists at least one PME in mixed strategy.

3.2. Pareto optimality. A pure PME allocation is Pareto optimal whenever it exists. This result is not trivial since an ME allocation under some $\omega \in A$ may not be Pareto optimal, even though it is Pareto optimal under $\omega$. Formally, we have the following Pareto optimality result.

Theorem 3.3. Assume (+Value) and (Quotal). Then, any pure PME object allocation is Pareto optimal.

Proof. Let $\omega$ and $\mu$ be the PME outcome of the first and second stages, respectively. Let $p$ be the price such that $(p, \mu)$ is an ME under $\omega$. Note first that no player in $N \backslash P$ can obtain any object due to (+Value) and the no-money assumption. So, we ignore $N \backslash P$. Suppose that $\eta \in \bar{A}$ Pareto dominates $\mu$.

It must be the case that $|\eta| \neq|\mu|$ holds; otherwise, $\mu$ cannot be a market equilibrium allocation. Then due to (+Value) and (Quota1), there exists $a \in O$ such that $\eta_{i}=a$ holds for some $i \in P$, and $\mu_{j} \neq a$ holds for all $j \in P$. Take such a tangible object $a$ and player $i$. Due to the genericity of the value profile, Pareto dominance implies that

$$
v_{i}\left(\eta_{i}\right)>v_{i}\left(\mu_{i}\right)
$$

holds. Note that nobody obtains $a$ on the equilibrium path of the PME. Thus, player $i$ could have profitably deviated from the PME by obtaining $a$ in the first stage. This is a contradiction. 
3.3. PME and DA allocations. This subsection presents two equivalence results about PME and DA allocations. Note first that the DA allocation is the unique stable allocation that Pareto dominates all the other stable allocations (see Lemma A.2 as well as Gale and Shapley (1962)). In other words, an allocation is stable and Pareto optimal if and only if it is the DA allocation that is Pareto optimal (the Pareto optimal DA allocation for short). Note also that in the single-stage model, the DA allocation becomes Pareto optimal for any value profile if and only if the priority profile is acyclical (Ergin (2002)). The first equivalence result states that the PME allocation with the truth-telling strategy profile is the Pareto optimal DA allocation for any value profile if and only if the priority profile is acyclical.

Even if the priority profile is acyclical, there may exist a pure PME of which allocation does not coincide with the DA allocation. This leads to the second equivalence result. It states that the Pareto optimal DA allocation is the unique pure PME allocation for any value profile if and only if the priority profile is unreversed.

First of all, the stability of object allocations is defined in the standard manner ${ }^{8}$.

Definition 3.1. An object allocation $\mu \in A$ is stable if

- $\forall i \in P, \forall j \in P\left[\mu_{j} \in O \wedge i>_{\mu_{j}} j \Rightarrow v_{i}\left(\mu_{i}\right) \geq v_{i}\left(\mu_{j}\right)\right]$,

- $\forall a \in O \forall i \in P\left[\left|\mu^{a}\right|<q^{a} \Rightarrow v_{i}\left(\mu_{i}\right) \geq v_{i}(a)\right]$,

- $\forall i \in P\left[v_{i}\left(\mu_{i}\right) \geq 0\right]$.

3.3.1. Acyclicity. We introduce the concept of priority cycle as stated in Ergin (2002) ${ }^{9}$.

Definition 3.2. A priority cycle consists of distinct $a, b \in O$ and $i, j, k \in N$ such that the following is satisfied:

$$
i>_{a} j>_{a} k>_{b} i \text {. }
$$

If the priority profile is not cyclical, it is called acyclical.

A generalized cycle of priority consists of distinct $a_{1}, a_{2}, \ldots, a_{n} \in O$ and $i, k_{1}, \ldots, k_{n} \in N$ such that the following are satisfied:

$$
k_{1}>_{a_{1}} i>_{a_{1}} k_{n}>_{a_{n}} k_{n-1}>_{a_{n-1}} k_{n-2} \ldots k_{2}>_{a_{2}} k_{1} .
$$

It is essentially shown that if $>$ has a generalized cycle, then it also has a cycle (Ergin (2002)).

We say that $\zeta^{*} \in \Sigma$ is a truth-telling strategy profile if for all $i \in P$ and for all $a, a^{\prime} \in \bar{O}$, $v_{i}(a)>v_{i}\left(a^{\prime}\right)$ implies that $a$ is ranked higher than $a^{\prime}$ in the list of player $i$. We call $\lambda\left(\zeta^{*}\right) \in A$ the DA allocation.

We then have the following equivalence result.

Theorem 3.4. Assume $|O| \geq 3,|N| \geq 3$, and (Quotal). Then, the following two statements are equivalent:

(1) For any $P$ with $|P| \geq 3$ and for all $v \in V_{+}$, the Pareto optimal DA allocation exists, and it is the PME allocation with the truth-telling strategy profile;

(2) $>$ is acyclical.

\footnotetext{
${ }^{8}$ Note that stability is defined for $A$ rather than $\bar{A}$. This suffices for our purpose since no allocation in $\bar{A} \backslash A$ is realized on the path of any pure PME due to (+Value).

${ }^{9}$ The definition of the cycle and acyclicity are different from that of Ergin (2002) in that Ergin (2002) includes the condition on scarcity in the definition as well.
} 
Proof. See Appendix A.3.

Theorem 3.4 states that the truth-telling strategy profile achieves the Pareto optimal DA allocation as a PME for any value profile if and only if the priority profile is acyclical. In the proof of (1) implying (2), assuming that the priority profile is cyclical, we construct $v$ and $P$ under which a player can profitably deviate from the truth-telling strategy.

Roughly speaking, the proof of (2) implying (1) goes as follows. We assume that there is no cycle in the priority profile, and that the truth-telling strategy profile does not constitute a PME allocation. Suppose that $\omega \in A$ is the first stage allocation under the truth-telling strategy profile. Then, there exists a player, denoted $i$, who can profitably deviate from the truth-telling strategy. From the property of DA and the strategy proofness of the truth-telling strategy profile, if player $i$ obtains a tangible object $a$ by deviation, player $i$ prefers $\omega_{i}$ to $a$. Therefore, player $i$ must trade in the second stage to be better off. Such a transaction is made possible only through a trading cycle. Note here that there is another player, player $j$, who is pushed out by player $i$ at $\omega_{j}=a$ due to $i$ 's deviation, i.e., player $i$ has higher priority than player $j$. Note also that there is yet another player, player $\ell$, who buys this object from player $i$ in the trading cycle. Player $\ell$ has lower priority than player $j$ if these players are distinct. Using the priority order of $i, j, \ell$ and the trading cycle, we construct a generalized cycle, which leads to a contradiction. The actual proof is lengthy since $j$ and $\ell$ may be the identical player, and in such a case, we have to construct a generalized cycle with distinct players in a recursive manner.

Some pure PME allocation may not coincide with the DA allocation even if the priority profile is acyclical. In the next subsection, we will discuss the equivalence condition under which the Pareto optimal DA allocation is the unique PME allocation.

3.3.2. Unreversedness. We are now in the position to present an equivalent condition under which the Pareto optimal DA allocation is the unique pure PME allocation. We call this condition the unreversed condition as defined in the following.

Top-two players, if any, are the two players who have higher priority than all the others at every object, i.e., $i$ and $j$ in $N$ such that for all $k \in N \backslash\{i, j\}$ and all $a \in O$, both $i>_{a} k$ and $j>_{a} k$ hold.

Definition 3.3. $>$ is reversed if either one of the following holds:

(1) there exist no top-two players;

(2) there exist top-two players, $i, j \in N$, and other players, $h, \ell \in N \backslash\{i, j\}$ such that $h>_{a} \ell$ and $\ell>_{b}$ h hold for some $a, b \in O$.

If $>$ is not reversed, it is said to be unreversed.

If the priority profile is unreversed, all the players except for the top-two players have the same priority order across the objects. Note that the unreversed priority profile is acyclical.

We then have the following result.

Theorem 3.5. Assume $|O| \geq 3,|N| \geq 3$, and (Quotal). Then the following two statements are equivalent:

(1) For any $P$ with $|P| \geq 3$ and for all $v \in V_{+}$, the Pareto optimal DA allocation exists, and it is the unique pure PME allocation;

(2) The priority profile $>$ is unreversed.

Theorem 3.5 states that there exists the unique pure PME allocation, and it is the Pareto optimal DA allocation for any value profile if and only if the priority profile is unreversed. In the proof 
of (1) implying (2), assuming that the priority profile is reversed, we construct $v$ and $P$ under which an unstable pure PME allocation is induced. An interesting case is that the priority profile is acyclical but reversed. Suppose that there are three participants in the mechanism, 1,2,3, and three objects, $x, y, z .1$ has the highest priority at all objects. 2 has higher priority at $x$ than 3 , but 3 has higher priority than 2 at $z$. Consider a value profile such that 1 likes $x$ most, and both 2 and 3 like $z$ most. In this case, 1 is indifferent between two different objects, $x$ and $z$, to obtain in the first stage. If 1 goes to $x$ in the first stage, 3 obtains $z$, and the final allocation becomes $(x, y, z)$, which is stable. However, if 1 obtains $z$ in the first stage, then 2 obtains $x$. In the second stage, 1 trades $z$ for $x$ with 2, who could have not obtained $z$ otherwise. This leads to $(x, z, y)$, an unstable PME allocation.

We show that (2) implies (1) by contradiction. Roughly speaking, the proof goes as follows. It is assumed that the priority profile is unreversed, but that there is an unstable PME allocation, $\mu$. It suffices to show that this induces a profitable deviation. Since $\mu$ is not stable, there exist players $i$ and $j$ in $P$ such that $i$ prefers $a=\mu_{j} \in O$ to $\mu_{i}$, but $j$ has lower priority than $i$ at $a$. This happens on the equilibrium path of the PME when $j$ obtains another object, $\omega_{j} \neq a$ in the first stage and trades it with $a$ in the second stage market. The owner $k$ of $a$ in the first stage must have higher priority than $i$ at $a$; otherwise, $i$ could have obtained it. Therefore, there exists a trading cycle that contains both $j$ and $k$ on the equilibrium path of this PME.

Note that if the priority profile is unreversed, all the players except top-two players are aligned from the higher priority to lower priority at all the objects. Therefore, no first stage deviation by $i$ changes the ownership of any player who has higher priority than $i$. This implies that $k$ still owns the same object $a$ after $i$ 's deviation. Also, since $j$ is in the same trading cycle as $k$, there exists at least one player whose priority is lower than $i$ in the trading cycle. It is verified that this enables player $i$ to obtain the object in the first stage from such a player and to trade it for $a$ with $k$. Hence, $i$ can profitably deviate from the PME. This leads to a contradiction. The actual proof is lengthy since it needs an inductive argument.

Proof.

$[(1) \Rightarrow(2)]$

\begin{tabular}{|c|c|c|c|}
\hline$i$ & 1 & 2 & 3 \\
\hline$v_{i}(x)$ & 30 & 20 & 10 \\
$v_{i}(y)$ & 20 & 10 & 20 \\
$v_{i}(z)$ & 10 & 30 & 30 \\
\hline
\end{tabular}

TABLE 3.1. Values

Take any priority profile $>$ that is reversed. First, consider the case where $>$ is cyclical. In this case, there exist distinct three players, say, 1,2,3, and distinct two objects $x, z$ such that $1>_{z} 3>_{z} 2>_{x}$ 1. There are three possible cases with respect to the priority order at $x$, (i) $2>_{x} 3>_{x} 1$, (ii) $3>_{x} 2>_{x} 1$, and (iii) $2>_{x} 1>_{x} 3$. We will see that for each of these three cases, 
the value profile $v$ given by Table 3.1 is a counter-example to the statement that the Pareto optimal DA allocation exists, and it is the unique pure PME allocation for $P=\{1,2,3\}$.

Case (i): $2>_{x} 3>_{x}$ 1. In this case, $(y, x, z)$ is the Pareto optimal DA allocation regardless of the priority order at $y$. There is a PME such that $(z, x, y)$ is the first stage outcome, and $(x, z, y)$ is the final allocation in the PME.

Case (ii): $3>_{x} 2>_{x}$ 1. In this case, $(y, x, z)$ is the Pareto optimal DA allocation regardless of the priority order at $y$. There is a PME such that $(z, y, x)$ is the first stage outcome, and $(x, y, z)$ is the final allocation.

Case (iii): $2>_{x} 1>_{x} 3$. In this case, $(y, x, z)$ is the Pareto optimal DA allocation regardless of the priority order at $y$. There is a PME such that $(z, x, y)$ is the first stage outcome, and $(x, z, y)$ is the final allocation.

Now, consider the case where the priority profile $>$ is acyclical but reversed. Again, assume without loss of generality that $1>_{z} 3>_{z} 2$ holds. Since $>$ is acyclical, $1>_{a} 2$ holds for all $a \in O$. Also, since $>$ is reversed, there must exist $x \in O$ such that $1>_{x} 2>_{x} 3$. Assume $P=\{1,2,3\}$. Then once again, the value profile $v$ given by Table 3.1 is a counter-example to the statement that the Pareto optimal DA allocation exists, and it is the unique pure PME allocation for $P=\{1,2,3\}$. Indeed, $(x, y, z)$ is the Pareto optimal DA allocation, while there is a PME such that $(z, x, y)$ is the first stage outcome, and $(x, z, y)$ is the final allocation.

$$
[(2) \Rightarrow(1)]
$$

Assume the priority profile is unreversed. Then, there are top-two players, denoted by 1 and 2. Now, write $N \backslash\{1,2\}=\{3,4, \ldots,|N|\}$. Assume without loss of generality that for $n, n^{\prime} \in$ $\{3,4, \ldots,|N|\}$ with $n<n^{\prime}, n>_{a} n^{\prime}$ holds for all $a \in O$.

Since $>$ is unreversed, it is acyclical. Theorem 3.4 implies that the Pareto optimal DA allocation exists, and it is a PME allocation. Thus, the set of PME allocations is nonempty.

Next, it is shown that any PME allocation is stable. Suppose $\gamma$ is an arbitrary PME allocation. Fix this $\gamma$ and the PME strategy that generates $\gamma$ in the sequel. Then, there exist a price vector $p$ and $\omega$ on the equilibrium path such that $(p, \gamma)$ is an ME under $\omega$.

Align $\left(p_{a}\right)_{a \in O^{\omega}}$ from the highest to the lowest: $p^{1}>p^{2}>\cdots>p^{\bar{s}}$. Let $O^{s} \subset O(s=1,2, \ldots, \bar{s})$ be the set of objects such that $p_{a}=p^{s}$ holds for $a \in O^{s}$. Also, given $i \in N$, define a set of objects $T^{i} \subset O$ to be the one of which elements are traded in the trading cycle that contains $\gamma_{i}$ on the equilibrium path of the PME. It is verified that $T^{i}$,s form an equivalence class, i.e., in particular, if $\gamma_{j} \in T^{i}$ holds for $j \in N$, then we have $T^{i}=T^{j}$. It is also verified that $T^{i} \cap O^{s} \neq \emptyset$ implies $T^{i} \subset O^{s}$.

To show the stability of $\gamma$, we consider the following claim $(\kappa=1,2, \ldots, \bar{s})$ :

(Claim $\kappa$ ) for all $i, j$ in $N$, if $\gamma_{i} \in O^{\kappa}, i>_{\omega_{j}} j$, and $\omega_{j}$ in $O^{s}$ with $s \leq \kappa-1$, then $i$ prefers $\gamma_{i}$ to any object in $T^{j} \subset O^{s}$.

If (Claim $\kappa$ ) holds for all $\kappa=1,2, \ldots, \bar{s}$, the PME allocation $\gamma$ is stable. For if not, there exist distinct $i$ and $j$ such that $i$ prefers $j$ 's object $\gamma_{j}$ while $i$ has higher priority than $j$ at $\gamma_{j}$. This happens only when the price of $\gamma_{j}$ is higher than that of $i$ 's endowment, $\omega_{i}$, and $j$ obtains $\gamma_{j}$ in the second stage market by exchanging it with his endowment $\omega_{j} \neq \gamma_{j}$. Otherwise, $i$ could have bought $\gamma_{j}$ or deprived $j$ of $\gamma_{j}$ on the path. Therefore, if a PME allocation $\gamma$ is not stable, the claim to be shown does not hold. This is a contraposition of what we will prove. 
We will prove (Claim $\kappa)(\kappa=1,2, \ldots, \bar{s})$ by induction. First of all, (Claim 1) trivially holds. Suppose next that (Claim $k^{\prime}$ ) holds for all $k^{\prime}=1, \ldots, k(k<\bar{s})$. We would like to prove (Claim $k+1)$.

Now, suppose the contrary, i.e., that there exist $i$ and $j$ in $N$ such that $\gamma_{i} \in O^{k+1}, i>_{\omega_{j}} j$, and $\omega_{j}$ in $O^{s}$ with $s \leq k$, but there exists $a \in T^{j}$ such that $v_{i}(a)>v_{i}\left(\gamma_{i}\right)$. Let $\ell \in N$ be the player who holds such an $a$, i.e., $\omega_{\ell}=a$. Since $\gamma$ is a PME allocation, $\ell>_{\omega_{\ell}} i$ holds; otherwise, $i$ would deprive $\ell$ of $\omega_{\ell}$ in the first stage.

We order the players who trade objects in $T^{j}$ in the following manner. First, let $\ell_{1}=\ell$. Next, let $\ell_{2}$ be the player who obtains $\gamma_{\ell_{1}}$ in the first stage, i.e., $\omega_{\ell_{2}}=\gamma_{\ell_{1}}$. This way, we order the players $\left(\ell_{1}, \ell_{2}, \ldots, \ell_{\left|T^{j}\right|}\right)$ with $\omega_{\ell_{1}}=\gamma_{\ell_{\mid T j}{ }^{j}}$. Thus, the trading cycle is given by

$$
\left(\left(\ell_{1}, \omega_{\ell_{1}}\right),\left(\ell_{2}, \omega_{\ell_{2}}\right), \ldots,\left(\ell_{\left|T^{j}\right|}, \omega_{\ell_{\mid T} j^{j}}\right),\left(\ell_{1}, \omega_{\ell_{1}}\right)\right) .
$$

Note that $j$ holds an object in $T^{j}$. Therefore, there exists at least one player whose priority is lower than $i$. Thus, if $i \in N^{k} \backslash\{1,2\}$, let $\ell_{h}$ be the first player in $\left(\ell_{1}, \ell_{2}, \ldots, \ell_{\left|T^{j}\right|}\right)$ with $\ell^{*}>i$. If $i=1$, then we have $\ell=2$ since $\ell>_{\omega \ell} i$ holds. Let $\ell_{h}=\ell_{2}$. Similarly, if $i=2$, let $\ell_{h}=\ell_{2}$.

In the first stage, $i$ can obtain $\ell_{h}$ 's object, and $i$ 's deviation changes the endowments of some players including $\ell_{h}$. Note that players $\ell_{1}, \ldots, \ell_{h-1}$ have higher priority than $i$ at their respective endowments.

It is verified that these players obtain the same objects even after $i$ 's deviation. To see this, first consider the case of $i \in N \backslash\{1,2\}$. In this case, the rejection chain aligns the players from the one with high priority to the one with low priority. Since the priority profile is unreversed, this rejection chain cannot put $i$ before $\ell_{h^{\prime}}$ who has higher priority at every object than anyone who in turn has lower priority at some object than $i$.

Next, consider the case of $i \in\{1,2\}$. Without loss of generality, suppose $i=1$. Note that any player in $N \backslash\{1,2\}$ cannot change 2's endowment as we have just shown above. Then, it is sufficient to verify that 1's deviation does not directly affect 2 . Note that $2>_{\omega_{2}} 1$ holds. This implies that 1 cannot change 2's endowment once 2 reaches $\omega_{2}$ in DA. Since 1 may have higher priority than 2 at other objects, it is possible that 2 is rejected by 1 and comes to $\omega_{2}$ on the equilibrium path. This is the only possible path where 2's endowment changes by 1's deviation. However, since 1 prefers $\omega_{2}$ to $\omega_{1}$, this could not happen on the equilibrium path toward $\gamma$. Indeed, if this is the case, 1 can strictly better off by taking $\omega_{2}$ without moving 2 . Therefore, any 1's deviation does not change 2's endowment, i.e., 2 gets the same $\omega_{2}$. The same argument holds when $i=2$.

Thus, players $\ell_{1}, \ldots, \ell_{h-1}$ end up with the same endowments after $i$ 's deviation. Remember that these players do not want to obtain an object in $O^{s}(s \leq k)$ that are held by a player with lower priority than them. Then, even if $i$ 's deviation changes the owners of objects in $O^{s}$ for some $s \leq k$, they do not want to obtain elements in such $O^{s}$. In other words, if one of players $\ell_{1}, \ldots, \ell_{h-1}$, say, $\ell_{h^{\prime}}$ prefers another object with a higher price than $p^{s}$, then the player who obtains this object has higher priority than $\ell_{h^{\prime}}$

Next, such player $\ell_{h^{\prime}}$ wants to buy the same object as in the case that $i$ does not deviate. As we have assumed, $\ell_{h^{\prime}}$ does not want to buy objects in $O^{t}$ with $t>k$. Indeed, if $\ell_{h^{\prime}}$ wishes to buy it, he/she would have bought it on the equilibrium path.

Combining the above arguments, even after $i$ 's deviation, the object obtained in the first stage by player $\ell_{h^{\prime}}$ with $h^{\prime}<h$ has the same price with $\omega_{\ell_{h}}$ that $i$ obtains through deviation. Therefore, by obtaining $\ell_{h}$ 's endowment, $i$ can create another trading cycle starting from $\left(\ell, \omega_{\ell}\right)$, reaches $\left(i, \omega_{\ell_{h}}\right)$, 
and comes back to $\left(\ell, \omega_{\ell}\right)$. This means that $i$ can exchange the depriving object with an object that is more preferable than $\gamma_{i}$. If $i$ can create another trading cycle and buy another object that is more preferable object than the one held by $\ell$, then this also becomes an ME. This means that $i$ can profitably deviate in either case. Therefore, $\gamma$ is not a PME allocation.

Thus, $($ Claim $\kappa)$ holds for all $\kappa=1,2, \ldots, \bar{s}$. Together with Theorem 3.3, this implies that $\gamma$ is a stable and Pareto optimal allocation. Hence, $\gamma$ is the Pareto optimal DA allocation. This completes the proof.

3.4. Relationship between single-stage models and the present two-stage model. The relationship between the present two-stage model and the standard single-stage DA may be summarized as follows.

First of all, acyclicity plays a key role in both models. On the one hand, a PME allocation is always Pareto optimal (Theorem 3.3) but may not necessarily be stable. The stability of the PME allocation with the truth-telling strategy profile is shown to be equivalent to the acycilicity of the priority profile (Theorem 3.4). Note that a stable allocation Pareto dominated by the DA allocation cannot be a pure PME allocation since every pure PME allocation is Pareto optimal.

On the other hand, the single-stage DA mechanism always induces a stable allocation by the truth-telling strategy profile (Gale and Shapley (1962)), but the allocation is Pareto optimal for any value profile if and only if the priority profile is acyclical (Ergin (2002)) ${ }^{10}$. This relationship is summarized in Table 1.1.

Next, Theorem 3.5 states that there exists the unique pure PME allocation, and it is the Pareto optimal DA allocation for any value profile if and only if the priority profile is unreversed. The DA allocation is the unique allocation achieved in the pure PME allocation.

There is a remark about Nash equilibrium in DA. In general, the single-stage DA has a Nash equilibrium that does not use the truth-telling strategy profile (see Table 3.2). In this economy, the single-stage DA has two classes of Nash equilibria in terms of allocation. The first class of equilibria is the one in which Player 1 (resp. 2) places $x$ (resp. y) on the top of the list. The equlibrium with the truth-telling strategy profile is contained in this class. The allocation is $(x, y)$. The second class of equilibria is the one in which Player 1 (resp. 2) places $y$ (resp. $x$ ) on the top of the list. The allocation of this type of equlibrium is $(y, x)$. Neither player has an incentive to deviate due to the priority profile. Note that $(y, x)$ cannot be a PME allocation (of the second stage) since the players would trade their goods with each other when their endowment profile is $(y, x)$.

\footnotetext{
${ }^{10}$ Ergin (2002) shows that the Pareto optimality of DA is equivalent not only to the acycilicity of the priority profile but also to the group strategyproofness. According to Takamiya (2001), the group strategyproofness is also equivalent to Maskin monotonicity. Kojima and Manea (2010) also shows more equivalence conditions in which Pareto optimality of allocations are achieved in DA.
} 


\begin{tabular}{|c|c|c|}
\hline$i$ & 1 & 2 \\
\hline$v_{i}(x)$ & 20 & 10 \\
\hline$v_{i}(y)$ & 10 & 20 \\
\hline
\end{tabular}

TABLE 3.2. Values and priority

One may wonder if the top trading cycle mechanism (TTC) as defined by Abdulkadiroğlu and Sönmez (2003) induces the same allocation as a PME allocation in the present two-stage economy. The answer is in the negative as Example 3.1 shows.

\section{Example 3.1.}

Let the pair of values and priority be given as follows.

\begin{tabular}{|c|c|c|c|c|lllll|}
\hline$i$ & 1 & 2 & 3 & 4 & & & & \\
\cline { 1 - 3 }$(x)$ & 40 & 20 & 40 & 10 & $4>_{x}$ & $2>_{x}$ & $3>_{x}$ & 1 \\
$v_{i}(y)$ & 20 & 40 & 30 & 20 & $1>_{y}$ & $3>_{y}$ & $2>_{y}$ & 4 \\
$v_{i}(z)$ & 30 & 30 & 20 & 30 & $4>_{z}$ & $3>_{z}$ & $2>_{z}$ & 1 \\
$v_{i}(w)$ & 10 & 10 & 10 & 40 & $1>_{w}$ & $4>_{w}$ & $2>_{w}$ & 3 \\
\hline
\end{tabular}

TABLE 3.3. Values and priority

In this example, a single-stage TTC results in $(x, z, y, w)^{11}$. On the other hand, the PME allocation of the two-stage economy under truth-telling strategy profile is $(z, y, x, w)$.

\section{The Economy with Money}

This section studies the economy with money. Efficiency is the main property that this section examines instead of Pareto optimality and stability studied in the previous section. If money is available, we have a very different economy from the one without money. Under the assumption of the quasi-linearity of utility functions, the definition of Pareto optimality is reduced to that of efficiency. Also, each player can be a buyer in the second stage even if he/she does not obtain an object in the first stage, and the market attains efficiency under the endowment profile at the beginning of the second stage. Then under the genericity assumption, the object allocation is uniquely determined no matter what the first stage assignment may be, provided that the total endowment of the second stage is unchanged. Thus, the priority profile of the first stage does not matter in terms of object allocation although it affects each player's eventual utility through money transfer. Hence, the stability notion plays little role in terms of the final allocation of the objects.

\footnotetext{
${ }^{11}$ TTC in this example is different from the one defined by Shapley and Scarf (1974) in that both players and objects point their favorite objects/players.
} 
4.1. Existence. First, we have the existence result of market equilibrium in the second stage due to Kaneko and Yamamoto $(1986)^{12}$.

Lemma 4.1. For all $\omega \in A$, there exists at least one market equilibrium under $\omega$.

If there exists a market equilibrium under every $\omega$, then by assigning a market equilibrium allocation under each $\omega$, we can construct a game for the first stage. Then a PME exists in the mixed strategy profile space since the existence of PME is reduced to the existence of Nash equilibrium. Thus, the following result is stated without proof.

Theorem 4.2. There exists at least one PME in mixed strategy.

4.2. Efficiency. We turn to the efficiency of PME. Note that even though the market attains efficiency under the endowment profile at the beginning of the second stage, efficiency may not be guaranteed when there are some leftovers that are not available in the second stage. Indeed, if there are not a sufficient number of players, we may not have efficiency. Let us consider the following example.

\section{Example 4.1.}

\begin{tabular}{|c|r|r|}
\hline$i$ & 1 & 2 \\
\hline$v_{i}(x)$ & 10 & 50 \\
$v_{i}(y)$ & 20 & 5 \\
\hline
\end{tabular}

TABLE 4.1. An inefficient PME

Suppose that the values together with $N$ and $O$ are given by Table 4.1, that $q^{x}=q^{y}=1$ holds, and that $1>_{a} 2$ holds for $a=x, y$. Then, there is a PME where only $x$ is consumed. We show it by construction. On the equilibriumm path of this PME, we let

$$
\omega=(x, \phi), p=\left(p_{x}, p_{y}\right)=(20,-), \mu=(\phi, x),
$$

and the utility gains of Players 1 and 2 are 20 and 30, respectively. Off the equilibrium path when 2 deviates to obtain the leftover $y$ in the first stage, we let

$$
\omega=(x, y), p=\left(p_{x}, p_{y}\right)=(40,10), \mu=(y, x) .
$$

If this is the case, then the utility gain of Player 1 is 50, while that of Player 2 is 20. Therefore, 2 has no incentive to deviate. The lack of incentives to deviate in other off-paths is straightforward.

If we add another player, 3, the situation changes even if this person is the lowest both in terms of priority and values. See Table 4.2. Also, suppose that $1>_{a} 2>_{a} 3$ holds for $a=x, y$. In

\footnotetext{
${ }^{12}$ The same proof due to Kaneko and Yamamoto (1986) can be applied to show this claim except for two modifications. First, Kaneko and Yamamoto (1986) assumes that the excess demand for a particular good is nonnegative if its price is zero, while the present analysis does not necessarily assume it. Instead, the present equilibrium notion allows the excess demand to be negative at the price of zero. Second, Kaneko and Yamamoto (1986) shows the existence of integral solution by using the unimodularity of the system of equations due to Hoffman and Kruskal (2010). The same proof is applicable to the present analysis by verifying the unimodularity of the system of equations of the present model, which is straightforward.
} 


\begin{tabular}{|c|r|r|r|}
\hline$i$ & 1 & 2 & 3 \\
\hline$v_{i}(x)$ & 10 & 50 & 3 \\
$v_{i}(y)$ & 20 & 5 & 3 \\
\hline
\end{tabular}

TABLE 4.2. An inefficient PME disappears

this case, an inefficient equilibrium similar to the one that existed before in the two player case disappears. To see this, consider the corresponding allocation, i.e.,

$$
\omega=(x, \phi, \phi), p=\left(p_{x}, p_{y}\right)=(20,-), \mu=(\phi, x, \phi) .
$$

This time, Player 3 obtains an object in neither stage. Thus, Player 3 's surplus is zero even though 3 has a positive value for the objects. Thus, 3 has an incentive to take the leftover. The above allocation cannot be an equilibrium outcome.

We generalize the argument of the above example to find a necessary and sufficient condition for efficiency. Note that $N$ and $>$ on $N$ are taken as given.

To begin with, given $\theta=1,2, \ldots$, let

$$
V_{\theta}=\left\{v \in V\left|\min _{a \in O}\right|\left\{i \in P \mid v_{i}(a)>0\right\} \mid=\theta\right\} .
$$

Given $v \in V_{\theta}, \min _{a \in O}\left|\left\{i \in P \mid v_{i}(a)>0\right\}\right|$ may be interpreted as the minimal demand. Roughly speaking, the following theorem states that a PME allocation is efficient for any value profile if and only if the minimal demand is sufficiently larger than $Q$, the sum of the quotas.

Theorem 4.3. Given $\theta=1,2, \ldots,|P|$, the following two statements are equivalent:

(1) for all $v \in V_{\theta}$, a pure PME exists, and every pure PME allocation is efficient;

(2) $\theta \geq 2 Q-\min _{a \in O} q^{a}$.

Roughly speaking, the proof of the theorem goes as follows. First, we prove that (1) implies (2). Suppose that the minimal demand is not sufficient so that (2) is violated. Given the priority profile, we would like to construct a value profile $v$ for which a pure PME allocation is not efficient. Inefficiency arises if there is a leftover in the first stage. First of all, we divide the players into two, sellers and buyers, in such a way that the sellers have higher priority than the buyers. Note that if the minimal demand is not so large, then all the players with positive values can become either a seller or a buyer. Next, $v$ is constructed in such a way that the sellers do not value the objects very much compared to the buyers. As a result, the sellers obtain some objects in the first stage and sell them in the second. In particular, they do not buy other objects in the second stage. The buyers obtain no object in the first stage and buy some in the second. This implies that there are two players, a seller and a buyer, for each tangible object. Since the minimal demand is not sufficiently large, if we attach the players to the objects in this manner, there must be some leftovers that are not available in the second stage. From these leftovers, some sellers would have gained some positive payoffs if they were obtained in the first stage by some "buyers." The buyers, however, would not do so; for if they do, they expect the price profile to change in an unfavorable manner. The existence of these leftovers is the source of inefficiency.

Next, we prove the opposite direction, i.e., (2) implies (1). Suppose that (2) holds. Suppose also that there is some leftover on the equilibrium path. Even if one tries to divide the players into 
sellers and buyers, there will be a player with a positive value who can be neither a seller nor a buyer. This is due to the fact that $\theta$ exceeds the threshold $2 Q-\min _{a \in O} q^{a}$. Then this player has an incentive to deviate and obtain a leftover. Therefore, no leftover would remain in the first stage. The rest is straightforward since a market equilibrium is efficient once all the objects are carried over to the second stage.

Proof. $[(1) \Rightarrow(2)]$

We prove this direction by contraposition. Take $\theta=1,2, \ldots,|P|$ as given. Assume $\theta<2 Q-$ $\min _{a \in O} q^{a}$. It suffices to show that there exist $v \in V_{\theta}$ and a pure inefficient PME. We prove this statement for the case of $\theta=|P|$. If $\theta<|P|$, we simply set the values of extra players to be negative at every tangible object and follow the same proof. Also, let all the values of the players in $N \backslash P$ be negative at every tangible object.

Write $O=\left\{a_{1}, \ldots, a_{\bar{L}}\right\}$ in such a way that $q^{a_{1}} \geq q^{a_{2}} \geq \ldots \geq q^{a_{\bar{L}}}$ holds. Note $|O| \geq 2$ and $q^{a_{\bar{L}}}=\min _{a \in O} q^{a}$. Note also that $q^{a_{1}}<|P|<2 Q-q^{a_{\bar{L}}}$ holds where the first inequality comes from the assumption in Subsection 2.2. Therefore, $|P|$ satisfies

$$
\sum_{\ell=1}^{L-1} 2 q^{a_{\ell}} \leq|P|<\sum_{\ell=1}^{L} 2 q^{a_{\ell}}
$$

for some $L=1, \ldots, \bar{L}$ where the left hand side is zero if $L=1$. Fix $L$. Let $O_{L}=\left\{a_{1}, \ldots, a_{L}\right\}$.

In constructing a pure inefficient PME, we first divide the players into sellers and buyers. To do this, we identify the players who have higher priority for the objects in $O_{L}$ than others. Let $S_{1}$ be the set of the top $q^{a_{1}}$ players in terms of priority at $a_{1}$ among $P$, i.e., $S_{1}=\left\{i_{1}, \ldots, i_{q^{a_{1}}}\right\}$ such that $i>_{a_{1}} j$ holds for all $i \in S_{1}$ and all $j \in P \backslash S_{1}$. Then sequentially define $S_{\ell}(\ell=2, \ldots, L-1)$ as the set of the top $q^{a_{\ell}}$ players in terms of priority at $a_{\ell}$ among $P \backslash\left[\cup_{\ell^{\prime}=1}^{\ell-1} S_{\ell^{\prime}}\right]$. As for $S_{L}$, define it as the set of top $\min \left\{q^{a_{L}},|P|-\sum_{\ell=1}^{L-1} 2 q^{a_{\ell}}\right\}$ players in terms of priority at $a_{L}$ among $P \backslash\left[\cup_{\ell^{\prime}=1}^{L-1} S_{\ell^{\prime}}\right]$. Let $S=\cup_{\ell=1}^{L} S_{\ell}$. Partition $P \backslash S$ into $B_{1}, \ldots, B_{L}$ in such a way that $\left|B_{\ell}\right|=q^{a_{\ell}}$ holds for $\ell=1, \ldots, L-1$, and $B_{L}=P \backslash\left[S \cup_{\ell=1}^{L-1} B_{\ell}\right]$. Note that $\left|B_{L}\right| \leq\left|S_{L}\right|$ holds, that $S_{L}$ may be empty, that $\left|S_{L}\right|<q^{a_{L}}$ implies $B_{L}=\emptyset$, and that $\left|B_{L}\right|<q^{a_{L}}$ holds.

Next, we construct $v$ as follows:

for $i \in S_{\ell}(\ell=1, \ldots, L-1)$, let $v_{i}(a)$ be any number in $(0,1)$ for all $a \in O$;

for $i \in B_{\ell}(\ell=1, \ldots, L-1)$, let $v_{i}(a)$ be any number satisfying the following:

$$
v_{i}(a) \in \begin{cases}(14,15) & \text { if } a=a_{\ell}, \\ (0,1) & \text { otherwise. }\end{cases}
$$

As for the players in $S_{L}$ and $B_{L}$, we define $v$ separately as follows. Partition $S_{L}$ into $S_{L}^{\prime}$ and $S_{L}^{\prime \prime}$ with $\left|S_{L}^{\prime}\right|=\left|B_{L}\right|$, and for $i \in S_{L}^{\prime}$, let $v_{i}(a)$ be any number in $(0,1)$ for all $a \in O$, for $i \in S_{L}^{\prime \prime} \cup B_{L}$,

$$
v_{i}(a) \in \begin{cases}(2,3) & \text { if } a=a_{L}, \\ (0,1) & \text { otherwise }\end{cases}
$$

Note that $S_{L}^{\prime}$ is empty if $B_{L}$ is empty. Note also that the above values are chosen in such a way that genericity is guaranteed. 
Then, let the first stage outcome be given by

$$
\omega_{i}^{*}= \begin{cases}a_{\ell} & \text { if } i \in S_{\ell},(\ell=1, \ldots, L), \\ \phi & \text { if } i \in B_{\ell},(\ell=1, \ldots, L) .\end{cases}
$$

To attain this profile, player $i \in S_{\ell}(\ell=1, \ldots, L)$ submits $a_{\ell}$ on top of the list. As for player $i \in B_{\ell}$ $(\ell=1, \ldots, L)$, he/she submits $a_{\ell}$ on top and $\phi$ on the second of the list.

In the second stage, let $p_{a_{\ell}}^{*}=10-\ell / L$ for all $\ell=1, \ldots, L-1$ and $p_{a_{L}}^{*}=1$ under $\omega^{*}$. Let the price vector be unchanged even if $i \in S$ unilaterally deviates. This is possible since the total endowment of the second stage does not change by such a deviation. Let $p_{a_{\ell}}(\ell=1, \ldots, L)$ change from $p_{a_{\ell}}^{*}$ to $p_{a_{\ell}}^{*}+1$ if $i \in B_{\ell}$ unilaterally deviates. Let other prices remain unchanged even after such a deviation.

Note that the price of the object other than $a_{1}, \ldots, a_{L}$ is at most one due to the construction of $v$. Assign any ME to other endowment profiles, i.e., nodes that are reached only when two or more players deviate. A typical behavior pattern and prices on the path are given in Table 4.3.

\begin{tabular}{|c|c|c|c|c|c|}
\hline & $a_{1}$ & $\cdots$ & $a_{L-1}$ & $a_{L}$ & leftovers \\
\hline & $B_{1}$ & & $B_{L-1}$ & $B_{L}$ & \\
\hline Buyers & $\overbrace{a}^{a \ldots a}$ & . & $\overbrace{\nabla+\ldots q}$ & 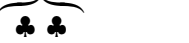 & \\
\hline \multirow{2}{*}{ Sellers } & $\Delta a \cdots$ & $\cdots$ & $\varphi \vee \cdots \varphi$ & $+2+2$ & 口व \\
\hline & $S_{1}$ & & $S_{L-1}$ & $S_{L}^{\prime}$ & \\
\hline price $p^{*}$ & $10-\frac{1}{L}$ & $\cdots$ & $10-\frac{L-1}{L}$ & 1 & \\
\hline
\end{tabular}

TABLE 4.3. A typical behavior pattern and prices on the path

We then show that the profile described above constitutes a PME. Observe first what the players do on the path:

- player $i \in S_{\ell}(\ell=1, \ldots, L-1)$ obtains object $a_{\ell}$ in the first stage, sells it to a player in $B_{\ell}$ at $p_{a_{\ell}}^{*}$ in the second stage, and gains $p_{a_{\ell}}^{*}=10-\ell / L$ through the two-stage activity;

- player $i \in B_{\ell}(\ell=1, \ldots, L-1)$ obtains $\phi$, the null object, in the first stage, buys $a_{\ell}$ in the second stage, and gains $v_{i}\left(a_{\ell}\right)-p_{a_{\ell}}^{*} \in(4,6)$;

- player $i \in S_{L}^{\prime}$ obtains object $a_{L}$ in the first stage, sells it to a player in $B_{L}$ at $p_{a_{L}}^{*}=1$, and gains 1;

- player $i \in S_{L}^{\prime \prime}$ obtains object $a_{L}$ in the first stage, consumes it, and gains $v_{i}\left(a_{L}\right) \in(2,3)$;

- player $i \in B_{L}$ obtains $\phi$ in the first stage, buys $a_{L}$ at $p_{a_{L}}^{*}=1$, and gains $v_{i}\left(a_{L}\right)-1 \in(1,2)$.

Next, we check what they obtain if they make a unilateral deviation:

- player $i \in S_{\ell}(\ell=1, \ldots, L-1)$ may obtain $a_{\ell^{\prime}}$ for some $\ell^{\prime}>\ell$ to gain $p_{a_{\ell^{\prime}}}^{*}, v_{i}(a) \in(0,1)$ for some $a \in \bar{O}$; all of them are less than $p_{a_{\ell}}^{*}$;

- player $i \in B_{\ell}(\ell=1, \ldots, L-1)$ may obtain a leftover, say, $a$ in the first stage and sell it and buy $a_{\ell}$ at the same time in the second stage. By doing so, he/she gains at most $v_{i}\left(a_{\ell}\right)+p_{a}-\left(p_{a_{\ell}}^{*}+1\right)$ due to a price increase of object $a_{\ell}$. Since $p_{a}$ is at most one, the gain is less than or equal to $v_{i}\left(a_{\ell}\right)-p_{a_{\ell}}^{*}$;

- since the prices of objects $a_{L}, \ldots, a_{\bar{L}}$ are at most one, player $i \in S_{L}^{\prime}$ gains at most one;

- player $i \in S_{L}^{\prime \prime}$ gains either one or $v_{i}\left(a_{L}\right)-1$; 
- player $i \in B_{L}$ may obtain a leftover, say, $a$ in the first stage and sell it and buy $a_{L}$ at the same time in the second stage. By doing so, he/she gains at most $v_{i}\left(a_{L}\right)+p_{a}-\left(p_{a_{L}}^{*}+1\right)$ due to a price increase of object $a_{L}$. Since $p_{a}$ is at most one, the gain is less than or equal to $v_{i}\left(a_{L}\right)-p_{a_{L}}^{*}$.

Hence, none of them has an incentive to make a unilateral deviation. Since there is a leftover that would have induced a positive utility gain, the PME is not efficient.

$[(1) \Leftarrow(2)]$

Assume $\theta \geq 2 Q-\min _{a \in O} q^{a}$.

Existence:

Let $\omega$ be an allocation with no leftover, i.e., $|\omega|=q$. Suppose $\left(p^{*}, \mu^{*}, m^{*}\right)$ is an ME under $\omega$ (such an ME exists). We may assume $p_{a}^{*}>0$ for $a \in O$ since there is a sufficient amount of demand for each $a \in O$. For any $\omega^{\prime}$ with no leftover, i.e., $\left|\omega^{\prime}\right|=q$, let $p\left(\omega^{\prime}\right)=p^{*}$. Adjusting $m^{\prime}$ appropriately, we obtain an $\operatorname{ME}\left(p^{*}, \mu^{*}, m^{\prime}\right)$ under $\omega^{\prime}$ since $|\omega|=\left|\omega^{\prime}\right|$ together with quasi-linearity implies that ME's under $\omega$ and $\omega^{\prime}$ are both efficient, allocation of which is uniquely determined by way of genericity (see, e.g., Shapley and Shubik (1971)).

Align the objects $O=\left\{a_{1}, a_{2}, \ldots, a_{L}\right\}$ in such a way that $p_{a_{1}} \geq p_{a_{2}} \geq \ldots, \geq p_{a_{L}}$ holds. Then, as in the proof of the other direction, let $S_{1}$ be the set of the top $q^{a_{1}}$ players in terms of priority at $a_{1}$ among $P$, i.e., $S_{1}=\left\{i_{1}, \ldots, i_{q^{a_{1}}}\right\}$ such that $i>_{a_{1}} j$ holds for all $i \in S_{1}$ and all $j \in P \backslash S_{1}$. Then sequentially define $S_{\ell}(\ell=2, \ldots, L)$ as the set of the top $q^{a_{\ell}}$ players in terms of priority at $a_{\ell}$ among $P \backslash\left[\cup_{\ell^{\prime}=1}^{\ell-1} S_{\ell^{\prime}}\right.$. Let $S=\cup_{\ell=1}^{L} S_{\ell}$.

Next, in the first stage, let

$$
\omega_{i}^{*}= \begin{cases}a_{\ell} & \text { if } i \in S_{\ell},(\ell=1, \ldots, L), \\ \phi & \text { if } i \in N \backslash S\end{cases}
$$

To attain this profile, the players in $S$ put what they are supposed to obtain at the top of the list to submit. The players in $P \backslash S$ submit the truth-telling strategies. Along the path, each player $i \in P$ obtains $\omega_{i}^{*}$ in the first stage. Moreover, even if one, say, player $i$, makes a unilateral deviation, there would be no leftover since there are players in $P \backslash S$ waiting for any leftover.

This strategy profile constitutes a pure PME along with the ME's mentioned above (and appropriately chosen ME's for other $\omega$ 's).

Efficiency:

Note that we have assumed $\theta \geq 2 Q-\min _{a \in O} q^{a}$. Take any $v \in V_{\theta}$. Suppose $a \in O$ has some leftover, i.e., $\left|\omega^{a}\right|<q^{a}$. Observe that at least $q^{a}$ players who cannot obtain $b \in O \backslash\{a\}$ in neither stage and have a positive value for $a$. Let $W$ be the set of such agents. Note $|W| \geq q^{a}>\left|\omega^{a}\right|$. Then $p_{a} \geq \min _{i \in W} v_{i}(a)>0$ holds; for if not, there would be excess demand for $a$. Then there exists $\ell \in W$ who obtains nothing in the first stage, i.e., $\omega_{\ell}=\phi$. This agent $\ell$ has an incentive to obtain the leftover in the first stage to gain $v_{\ell}(a)$ instead of $\max \left\{v_{\ell}(a)-p_{a}, 0\right\}$. Thus, there is no leftover. Once this is established, we resort to the efficiency property of the second stage market equilibrium to assure efficiency. 


\section{College Admission and Labor Market}

This section studies the economy where players are divided into two groups, sellers and buyers $^{13}$. This analysis may be interpreted as a college admission problem followed by a labor market: the objects are college degrees, the sellers are students, and the buyers are firms. The first stage is a many-to-one matching between students and colleges. In the second stage, a job market opens, and each firm hires at most one student based on his/her college degree.

Let $P$ be the set of students and $N \backslash P$ be the set of firms. College degrees are objects. A firm can demand a degree only if it is owned by some student ${ }^{14}$. Every student selects a college (including not going to college, corresponding to $\phi$ ), taking into account the future job prospect.

We assume the following.

(NP): $v_{i}(a) \leq 0$ holds for all $i \in P$ and all $a \in \bar{O}$,

Assumption (NP) implies that only the firms, not the students, intrinsically demand the college degrees.

5.1. Existence. The following existence result is proven by Kaneko and Yamamoto (1986).

Lemma 5.1. (Kaneko and Yamamoto (1986)) Assume (NP). Given $\omega \in$ A, there exists at least one market equilibrium under $\omega$.

Then a PME exists in mixed strategy since the existence of PME is reduced to the existence of Nash equilibrium. The following result is stated without proof.

Theorem 5.2. Assume (NP). Then there exists at least one PME.

5.2. Efficiency. As in the previous section, efficiency is the main property examined here. First of all, under the assumption of the quasi-linearity of utility functions, the definition of Pareto optimality is reduced to that of efficiency. Next, the priority profile of the first stage no longer matters in terms of object allocation, and therefore, the stability notion plays little role in terms of the final allocation of the objects.

In order to state the subsequent result, we need to modify the definition of minimal demand from what we have in the previous section. Given $\theta=1,2, \ldots$, let

$$
V_{\theta}=\left\{v \in V\left|\min _{a \in O}\right|\left\{i \in N \backslash P \mid v_{i}(a)>0\right\} \mid=\theta\right\} .
$$

The next theorem corresponds to Theorem 4.3.

Theorem 5.3. Assume (NP). Given $|P| \geq Q$ and $\theta=1, \ldots,|N \backslash P|$, the following two statements are equivalent:

(1) for all $v \in V_{\theta}$, a pure PME exists, and every pure PME allocation is efficient;

(2) $\theta>Q$.

Note that Condition (2) of this theorem is different from Condition (2) of Theorem 4.3. This difference is due to the fact that the sellers and the buyers have already been separated as the students and the firms in the present section.

\footnotetext{
${ }^{13}$ Note that in the proof of Theorem 4.3, the players are divided into the sellers and the buyers in the construction of PME. But, the division is endogenous. While the separation between sellers and buyers is exogenously determined in the present section.

${ }^{14} \mathrm{We}$ do not consider signaling effects here.
} 
A rough idea of the proof is similar to that of Theorem 4.3. Indeed, in proving that (1) implies (2), we suppose that the minimal demand is not sufficient, i.e., (2) is violated. The construction of the value profile is easier in the present proof than in the previous one since there is no need to worry about the buyers trying to obtain the objects in the first stage, which is prohibited as an outset.

Note also that the sellers do not value tangible objects. A seller has a strict incentive to obtain an object only if its price is expected to be positive. We construct an ME where the price of some tangible object is zero even if it induces a positive payoff for some buyer. The sellers may not obtain such an object, which leads to the existence of a leftover, and therefore, inefficiency.

As for the proof of (2) implying (1), we first show the existence. Under Condition (2), the minimal demand is sufficiently large so that every price of tangible object is positive on the equilibrium path of any pure PME. Whenever all the tangible objects may have positive prices, efficiency will be attained since there is no leftover.

In the following proof of Theorem 5.3, we skip some details when they are similar to what we have stated in the proof of Theorem 4.3.

Proof. $[(1) \Rightarrow(2)]$

Assume $\theta \leq Q$. We construct $v \in V_{\theta}$ as follows. Align the objects in an arbitrary manner, $O=\left\{a_{1}, \ldots, a_{\bar{L}}\right\}$. There exists a unique $L=1, \ldots, \bar{L}$ such that $q_{a_{1}}+\cdots+q_{a_{L-1}}<\theta \leq q_{a_{1}}+\cdots+q_{a_{L}}$ holds. Fix $L$. Let $N_{f} \subset N \backslash P$ satisfy $\left|N_{f}\right|=\theta$, and let $v_{i}(a)<0$ for all $a \in O$ and all $i \notin N_{f} \cup P$. Then assign a number to each $v_{i}(a)\left(i \in N_{f}, a \in O\right)$ in such a way that for each $\ell=1, \ldots, \bar{L}-1$, and for all $i, j \in N_{f}, v_{i}\left(a_{\ell}\right)>v_{j}\left(a_{\ell+1}\right)>0$ holds.

Let $\mu^{*}$ be the efficient object allocation given $v$. It must be the case that $\left|\mu^{* a}\right|=q^{a}$ for $a=$ $a_{1}, \ldots, a_{L-1}$ and that $0<\left|\mu^{* a_{L}}\right| \leq q^{a_{L}}$. Consider $\omega$ with $|\omega|=\left|\mu^{*}\right|$. Then $\left(p, \mu^{*}, m\right)$ becomes an ME under $\omega$ for some $p$ and $m$. It is verified, due to the way we construct $v$, that $p_{a_{1}} \geq p_{a_{2}} \geq \ldots \geq p_{a_{L}}$. Then there is another ME, denoted $\left(p^{*}, \mu^{*}, m^{*}\right)$, such that $p_{a_{\ell}}^{*}=p_{a_{\ell}}-p_{a_{L}}$ holds for all $\ell=1, \ldots, L$. Note that $p_{a_{L}}^{*}=0$ holds.

Assign the objects to the players in $P$ in the first stage from $a_{1}$ to $a_{L-1}$ to fill their respective quotas, using $>$, i.e., those who have higher priority at $a_{1}$ obtain $a_{1}$, and so on. As for $a_{L}$, assign the objects to the remaining students so that the total number of the students assigned to some tangible objects becomes $\theta$. Assign the other students to $\phi$. Denote this assignment profile $\omega^{*}$. Let $\left(p^{*}, \mu^{*}, m^{\prime}\right)$ be the ME under any $\omega^{\prime}$ with $\left|\omega^{\prime}\right|=\left|\omega^{*}\right|$. The existence of such an ME is proven in the same manner as in Shapley and Shubik (1971).

Take one player, say, $i \in P$, who obtains $a_{L}$ under $\omega^{*}$. Change $\omega_{i}^{*}$ to $\phi$ and obtain $\omega^{* *}$, i.e., for all $j \neq i, \omega_{j}^{* *}=\omega_{j}^{*}$ holds. We would like to have this $\omega^{* *}$ as a PME allocation of the first stage. To attain this profile, the players in $P$ put what they are supposed to obtain at the top of the list to submit. Let us check if there is no incentive to deviate. Under $\omega^{* *}$, there is one firm in $N_{f}$ that cannot buy a tangible object in the second stage, and there is at least one student who does not obtain a leftover in the first stage. If such a student obtains $a_{L}$, then the first stage object allocation becomes $\omega^{\prime}$ with $\left|\omega^{\prime}\right|=\left|\omega^{*}\right|$, and therefore, the price of $a_{L}$ is zero. Note that the price of object $\ell>L$ that this student obtains by deviation can be zero in equilibrium since he/she obtains an object of which value is less than $a_{L}$. Thus, the student has no incentive to deviate in the first stage. An inefficient outcome arises as a PME allocation.

$[(1) \Leftarrow(2)]$ 
Suppose $\theta>Q$. Take $v \in V_{\theta}$ as given.

We show existence first. Construct $\omega^{*}$ as follows. Take some $\omega$ with $|\omega|=q$. Let $\left(p^{*}, \mu^{*}, m\right)$ be an ME under $\omega$. Align $O=\left\{a_{1}, \ldots, a_{L}\right\}$ in such a way that $p_{a_{1}}^{*} \geq p_{a_{2}}^{*} \geq \ldots \geq p_{a_{L}}^{*}$ holds. Since $\theta>Q$ holds, there exists $j \in N \backslash P$ such that $\mu_{j}^{*}=\phi$ and $v_{j}\left(a_{L}\right)>0$ hold. Therefore, we have $p_{a_{L}}^{*} \geq v_{j}\left(a_{L}\right)>0$. Assign objects $a_{1}, \ldots, a_{L}$ to the players in $P$ in the first stage, using $>$, i.e., assign $a_{1}$ to the top $q^{a_{1}}$ students in terms of priority and continue this process up to object $a_{L}$. Assign the other students, if any, to $\phi$. Denote this assignment profile $\omega^{*}$. Under $\omega^{*},\left(p^{*}, \mu^{*}, m^{*}\right)$ becomes an ME for some $m^{*}$. Let $\omega^{*}$ be the outcome of the first stage. Then together with appropriate off-path ME's, we have a PME as nobody has an incentive to deviate.

We next prove efficiency. Suppose that $(\sigma,(p(\omega), \mu(\omega), m(\omega)))$ is a PME. Take any $\omega$. Since $\theta>Q$ holds, for all $a \in O$, there exists $j \in N \backslash P$ such that $\mu_{j}(\omega)=\phi$ and $v_{j}(a)>0$ hold. Therefore, $p_{a}(\omega) \geq v_{j}(a)>0$ for all $a \in O$; otherwise, $j$ would buy $a$ in an ME. Let $\omega^{*}=\lambda(\sigma)$. Suppose that $a \in O$ has some leftover, i.e., $\left|\omega^{* a}\right|<q^{a}$. Since $|P| \geq Q$, there exists at least one student who does not obtain any tangible object in the first stage under $\omega^{*}$. This player has an incentive to obtain the leftover $a$ since under any $\omega, p_{a}(\omega)>0$ as we have shown.

\section{Conclusion}

The present paper has proposed a new framework, by explicitly formulating a two-stage model where indivisible objects are allocated through the first stage matching mechanism and traded in the second stage market. As the first stage matching mechanism, we have used the deferred acceptance algorithm (DA). This framework is applicable to both non-monetary and monetary markets in the second stage. The notion of perfect market equilibrium (PME) has been defined according to which market equilibrium prevails in the second stage both on and off the path, and Nash equilibrium is played in the first stage game induced by these second stage outcomes.

We have set forth the equivalence conditions where stability and efficiency are attained in equilibrium. In the case of non-monetary market, it has been shown that any pure PME allocation is always Pareto optimal, while the PME allocation with the truth-telling strategy profile is stable if and only if the priority profile is acyclical. Also, it has been shown that the Pareto optimal DA allocation is the unique pure PME allocation if and only if the priority profile is unreversed.

In the case of monetary market, the question is whether the PME allocations always attain efficiency or not. We have considered two cases. First, we have considered the economy in which the players may become buyers and sellers at the same time. The minimal demand is key to the equivalence condition. If it is greater than a certain threshold, all the PME allocations are efficient. Next, we have considered the economy in which players are divided into two groups, sellers and buyers. Again, if the minimal demand is greater than another certain threshold, all the PME allocations are efficient.

The present analysis is limited in various respects. To begin with, while DA is used as the first stage mechanism, it may be modified to incorporate general matching mechanisms. Second, the present paper uses a market as the second stage decentralized mechanism. The players may negotiate in a decentralized manner. Shapley value and noncooperative bargaining procedures may be adopted as the second stage decentralized mechanism. Third, it is assumed that each player obtains only one unit of tangible objects. The analysis may be extended to the case in which multiple objects can be traded in the second stage. Fourth, quasi-linearity is often a strong 
assumption. This assumption may be weakened to see what happens. Fifth, sometimes, payoffs may be induced by holding the object in the first stage. An example would be the economy in which students directly obtain utility from their degree. All of these variations remain for the future research. 


\section{Appendix A. No Money}

\section{A.1. Examples: Quotas and values.}

Existence of ME is not guaranteed if the quota exceeds one for some object type as the next example shows.

\section{Example A.1.}

\begin{tabular}{|c|r|r|r|}
\hline$i$ & 1 & 2 & 3 \\
\hline$v_{i}(x)$ & 10 & 20 & 20 \\
$v_{i}(y)$ & 20 & 10 & 10 \\
\hline
\end{tabular}

TABLE A.1. Values

Let the values of this economy be given in Table A.1. Suppose

$$
\omega=(x, y, y) \text {. }
$$

Then we have no market equilibrium in the second stage under $\omega$. To begin with, we have $p_{x} \leq 20$ and $p_{y} \leq 10$. For if not, there would be excess supply with a positive price. Consider two cases. First, suppose $p_{x} \leq p_{y} \leq 10$. Then both Players 2 and 3 can afford $x$, and therefore, the demand for $x$ is at least two, which leads to excess demand as there is only one unit of object $x$. Second, suppose $p_{x}>p_{y}$. Then no player demands $x$, which leads to excess supply for $x$ with a positive price. Thus, no market equilibrium exists.

Also, existence is not guaranteed if (+Value) is violated.

\section{Example A.2.}

\begin{tabular}{|c|r|r|r|}
\hline$i$ & 1 & 2 & 3 \\
\hline$v_{i}(x)$ & 20 & -10 & 20 \\
$v_{i}(y)$ & 10 & -20 & 10 \\
\hline
\end{tabular}

TABLE A.2. Values

Let the values of this economy be given in Table A.2. Suppose

$$
\omega=(\phi, x, y) \text {. }
$$

Then we have no market equilibrium in the second stage under $\omega$. Suppose the contrary, i.e., that $p$ is a market equilibrium price. First, we have $p_{\phi}=0$. Next, we would like to show $p_{x}=0$. Suppose not, i.e., $p_{x}>0$. Then there must be a positive demand for $x$, which occurs only if $p_{y} \geq p_{x}>0$ since Player 3 must demand $x$. This implies that there is no demand for $y$ since Player 1 has neither money nor object with a positive price. This is a contradiction. Thus, $p_{x}=0$ holds. But, this would induce the excess demand for $x$. Hence, no market equilibrium exists. 


\section{A.2. Lemmata and proofs: PME and DA allocations.}

Lemma A.1. Assume (+Value), and (Quota1). There exists a stable and Pareto optimal allocation if the priority profile is acyclical. Moreover, if $\mu$ is stable and Pareto optimal, there exists a price vector $p$ such that $(p, \mu)$ is an ME given $\mu$ itself as an endowment profile.

Proof. Assume (+Value), and (Quota1). In the sequel, we disregard the players in $N \backslash P$ because they cannot buy any object even in the second stage. Thus, without loss of generality, consider the case of $P=N$. Also, suppose that the priority profile is acyclical. Then, Ergin (2002) shows that the DA allocation $\mu$ becomes a stable and Pareto optimal allocation.

First, we assign a price to each object $a$ using the trading cycle mechanism with an initial object profile $\mu$ (see Shapley and Scarf (1974) for the definition of the trading cycle mechanism). Let $\left(p_{a}\right)_{a \in O}$ be such a constructed price profile. Then, no player has an incentive to trade in the second stage under $\mu$. Therefore, $(p, \mu)$ is a market equilibrium under $\mu$ itself.

Lemma A.2. (Gale and Shapley (1962),Theorem 2) Assume (+Value) and (Quota1). Let $\eta \in A$ be the allocation attained by the truth-telling strategy profile in the single-stage model. Suppose also that $\mu \in A$ with $\mu \neq \eta$ is stable. Then, $\mu$ is Pareto dominated by $\eta$.

The following corollary is a direct consequence of the above lemma, which is stated without proof.

Corollary A.3. Assume (+Value) and (Quotal). If there exists a stable and Pareto optimal allocation, it is unique and coincides with the Pareto optimal DA allocation.

\section{A.3. Proof of Theorem 3.4.}

Now we are in the position to state the proof of Theorem 3.4.

Proof.

$[(1) \Rightarrow(2)]$

This is a proof by contraposition. Suppose that the priority has a cycle, i.e., for distinct objects $x, y \in O$ and distinct players $1,2,3 \in N, 1>_{x} 2>_{x} 3>_{y} 1$ holds.

Now consider the case of $P=\{1,2,3\}$. Also, consider the following $v \in V_{+}{ }^{15}$.

\begin{tabular}{|c|c|c|c|}
\hline$i$ & 1 & 2 & 3 \\
\hline$v_{i}(x)$ & 10 & 30 & 30 \\
$v_{i}(y)$ & 30 & 10 & 20 \\
$v_{i}(z)$ & 20 & 20 & 10 \\
\hline
\end{tabular}

TABLE A.3. Values

\footnotetext{
${ }^{15}$ Although genericity is violated in this $v$, the argument does not depend upon non-genericity.
} 
Given these $P$ and $v$, the Pareto optimal DA allocation is $(z, x, y)$. However, Player 1 has an incentive to deviate from the truth-telling strategy and obtain $x$ in the first stage. By deviation, 1 can always trade $x$ with $y$ in the second stage. There is no PME where 1 does not obtain $y$ after the market exchange. Thus, this Pareto optimal DA allocation cannot be achieved as a PME object allocation.

$[(1) \Leftarrow(2)]$

Assume (Quota1). Assume that there is no cycle in the priority. Lemma A.1 implies that for each $P \subset N$ and $v \in V_{+}$, there exists a stable and Pareto efficient allocation. Suppose the contrary, i.e., there exist $P \subset N$ and $v \in V_{+}$such that a stable and Pareto optimal object allocation $\mu$ is not a PME allocation. What we would like to show is the existence of a priority cycle under this contrapositive assumption.

First, we disregard the players in $N \backslash P$ because they cannot buy any object even in the second stage. Thus, without loss of generality, consider the case of $P=N$. Next, we construct a profile of ME's. Since $\mu$ is Pareto optimal, there exists a price vector $p$ such that $(p, \mu)$ is an ME under $\mu$ as an endowment profile. Also, by Lemma 3.1, for any endowment profile $\omega$, there exists an ME. Let $(p(\omega), \mu(\omega))_{\omega \in A}$ be a profile of market equilibrium that satisfies $(p(\mu), \mu(\mu))=(p, \mu)$.

Let $\zeta^{*}=\left(\zeta_{j}^{*}\right)_{j \in N}$ be the truth-telling strategy profile. Then, the outcome of the first stage is $\mu$ under $\zeta^{*}$. Since $\left(\zeta^{*},(p(\omega), \mu(\omega))_{\omega \in A}\right)$ is not a PME, there exist $i \in N$ and $\zeta_{i}$ such that

$$
\mathbf{E}\left[u_{i}(\cdot) \mid\left(\zeta_{i}, \zeta_{-i}^{*}\right)\right]>\mathbf{E}\left[u_{i}(\cdot) \mid \zeta^{*}\right]
$$

holds. Fix this player $i$ throughout the proof.

Let $\omega^{*}=\lambda\left(\zeta^{*}\right)$ and $\hat{\omega}=\lambda\left(\zeta_{i}, \zeta_{-i}^{*}\right)$. We have $\omega^{*} \neq \hat{\omega}$; otherwise $i$ cannot be better off. DA and strategy proofness of $\zeta^{*}$ imply $v_{i}\left(\omega_{i}^{*}\right) \geq v_{i}\left(\hat{\omega}_{i}\right)$ and $i$ must trade through a trading cycle in the second stage to be better off. Let $\left(\left(k_{0}, \hat{\omega}_{0}\right),\left(k_{1}, \hat{\omega}_{k_{1}}\right), \ldots,\left(k_{\bar{n}}, \hat{\omega}_{k_{\bar{n}}}\right)\right)$ with $k_{0}=k_{\bar{n}}=i$ and $\hat{\omega}_{0}=\hat{\omega}_{k_{\bar{n}}}$ be the corresponding trading cycle:

$$
\begin{aligned}
& v_{k_{n}}\left(\hat{\omega}_{k_{n+1}}\right)>v_{k_{n}}\left(\hat{\omega}_{k_{n}}\right) ; \\
& k_{n+1}>_{\hat{\omega}_{k_{n+1}}} k_{n}, \quad n=0,1, \ldots, \bar{n}-1
\end{aligned}
$$

Also, note that $k_{1}, \ldots, k_{\bar{n}}$ are all distinct players; otherwise these players do not constitute a trading cycle.

Now, we consider an auxiliary situation by running DA without $i$ at first, and after this algorithm is tentatively terminated, we put player $i$ in the algorithm and continue the process until it stops. Note that DA object allocation is not affected by the order of moves as discussed in Dubins and Freedman $(1981)^{16}$. Player $i$ obtains $\hat{\omega}_{i}$ by rejecting another player, say, $\ell_{1}$; otherwise, i.e., if $\hat{\omega}_{i}$ had not been occupied by some player, then player $k_{\bar{n}-1}$ would have gotten it instead of object $\omega_{k_{\bar{n}-1}}$ due to (A.2). Also, there is a chain of rejection $\ell_{1}, \ell_{2}, \ldots, \ell_{R}$ where $\ell_{r+1}$ is rejected by $\ell_{r}(r=$ $1, \ldots, R-1)$, and $\ell_{R}$ ends up with either a leftover or $\phi$. Note that all the objects $\hat{\omega}_{\ell_{0}}, \hat{\omega}_{\ell_{1}}, \ldots, \hat{\omega}_{\ell_{R}}$ are distinct; otherwise, there is a priority cycle.

If $\ell_{1}$ is distinct from $k_{n}$ for all $n=1, \ldots, \bar{n}$, then recalling $k_{0}=k_{\bar{n}}=i$, we have

$$
i>_{\hat{\omega}_{i}} \ell_{1}>_{\hat{\omega}_{i}} k_{\bar{n}-1}>_{\hat{\omega}_{k_{\bar{n}}-1}} k_{\bar{n}-2}>_{\hat{\omega}_{k_{\bar{n}}-2}} \cdots>_{\hat{\omega}_{k_{2}}} k_{1}>_{\hat{\omega}_{k_{1}}} k_{0}=i
$$

which is a generalized cycle. A contradiction. Thus, $\ell_{1}=k_{n_{1}}$ for some $n_{1}=1, \ldots, \bar{n}$.

\footnotetext{
${ }^{16}$ DA discussed in this auxiliary situation is essentially the same as the one defined in Dubins and Freedman (1981).
} 
Using exactly the same argument, i.e., using $\ell_{1}$ and $\ell_{2}$ in place of $i$ and $k_{n_{1}}$, we conclude that if $\ell_{2}$ is distinct from $k_{n}$ for all $n=1, \ldots, n_{1}$, then there exists a generalized cycle:

$$
i>\hat{\omega}_{i} \ell_{1}>\hat{\omega}_{k_{n_{1}}} \ell_{2}>_{\hat{\omega}_{k_{n_{1}}}} k_{n_{1}}>_{\hat{\omega}_{k_{n_{1}}-1}} \cdots>_{\hat{\omega}_{k_{2}}} k_{1}>_{\hat{\omega}_{k_{1}}} k_{0}=i \text {. }
$$

This process ends in at most $\bar{n}$ steps.

By induction, the rejection chain reaches $k_{1}$. Then, for some $r, \ell_{r}=k_{1}$. Note that there is $\ell_{r+1}$ who is rejected by $\ell_{r}$ at $\omega_{k_{1}}$. Then, there is a generalized cycle:

$$
\ell_{r}>_{\omega_{k_{1}}} \ell_{r+1}>_{\omega_{k_{1}}} i>_{\omega_{k_{0}}} \ell_{1}>_{\omega_{k_{n_{1}}}} \cdots>_{\omega_{k_{2}}} \ell_{r}
$$

Finally, the rejection chain does not reach $k_{0}$ because the objects are distinct. This leads to a contradiction. 


\section{REFERENCES}

AbdulkadiroĞLu, A., AND T. Sönmez (2003): "School choice: A mechanism design approach,” American economic review, 93(3), 729-747.

Coase, R. (1960): “The Problem of Social Cost,” JL E Econ., 3, 1.

Crawford, V. P., ANd E. M. Knoer (1981): "Job matching with heterogeneous firms and workers," Econometrica: Journal of the Econometric Society, pp. 437-450.

Demsetz, H. (1964): "The exchange and enforcement of property rights," The Journal of Law and Economics, 7, 11-26.

Dubins, L. E., AND D. A. Freedman (1981): "Machiavelli and the Gale-Shapley algorithm," The American Mathematical Monthly, 88(7), 485-494.

ERGIN, H. I. (2002): "Efficient resource allocation on the basis of priorities," Econometrica, 70(6), 2489-2497.

Gale, D., AND L. S. Shapley (1962): "College admissions and the stability of marriage," The American Mathematical Monthly, 69(1), 9-15.

Hafalir, I., AND V. Krishna (2008): “Asymmetric auctions with resale,” American Economic Review, 98(1), 87-112.

Hoffman, A. J., AND J. B. Kruskal (2010): "Integral boundary points of convex polyhedra," in 50 Years of Integer Programming 1958-2008, pp. 49-76. Springer.

Jehiel, P., AND B. Moldovanu (1999): "Resale markets and the assignment of property rights," The Review of Economic Studies, 66(4), 971-991.

Kaneko, M. (1982): “The central assignment game and the assignment markets," Journal of Mathematical Economics, 10(2-3), 205-232.

Kaneko, M., AND Y. Yамамото (1986): "The existence and computation of competitive equilibria in markets with an indivisible commodity," Journal of Economic Theory, 38(1), 118-136.

Kojima, F., AND M. Manea (2010): “Axioms for deferred acceptance,” Econometrica, 78(2), 633-653.

Maskin, E., AND J. Moore (1999): “Implementation and renegotiation,” Review of Economic studies, pp. 39-56.

Rотн, A. E. (1982): "The economics of matching: Stability and incentives," Mathematics of operations research, 7(4), 617-628.

Roth, A. E., AND M. SотомаYor (1989): “The college admissions problem revisited," Econometrica: Journal of the Econometric Society, pp. 559-570.

ShaPley, L., AND H. SCARF (1974): “On cores and indivisibility,” Journal of mathematical economics, 1(1), $23-37$.

Shapley, L. S., AND M. Shubik (1971): “The assignment game I: The core,” International Journal of game theory, 1(1), $111-130$

Sotomayor, M. (2008): "The stability of the equilibrium outcomes in the admission games induced by stable matching rules," International Journal of Game Theory, 36(3), 621-640.

ТАкаміYа, K. (2001): "Coalition strategy-proofness and monotonicity in Shapley-Scarf housing markets," Mathematical Social Sciences, 41(2), 201-213.

Wako, J. (1984): "A note on the strong core of a market with indivisible goods," Journal of Mathematical Economics, 13(2), 189-194.

Faculty of Economics, University of Tokyo, 7-3-1 Hongo, Bunkyo-ku, Tokyo 113-0033, JAPAN.

E-mail address: amatsui@e.u-tokyo.ac.jp

URL: http://www.e.u-tokyo.ac.jp/ amatsui

Department of Economics, Northwestern University, 2211 Campus Drive, Evanston, IL 60208, U.S.A. 